\title{
Mathematical Analysis of Control Strategies of HCV in a Community with Inflow of Infected Immigrants
}

\author{
Neterindwa Ainea ${ }^{*}$, Estomih S. Massawe ${ }^{1}$, Oluwole Daniel Makinde ${ }^{2}$, Lucy Namkinga ${ }^{3}$ \\ ${ }^{1}$ Mathematics Department, University of Dar es Salaam, Dar es Salaam, Tanzania \\ ${ }^{2}$ Faculty of Military Science, Stellenbosch University, Cape Town, South Africa \\ ${ }^{3}$ Department of Molecular Biology and Biotechnology, University of Dar es Salaam, Dar es Salaam, Tanzania \\ Email: ${ }^{*}$ neteainea@gmail.com
}

Received 24 January 2015; accepted 15 February 2015; published 16 February 2015

Copyright (C) 2015 by authors and Scientific Research Publishing Inc.

This work is licensed under the Creative Commons Attribution International License (CC BY). http://creativecommons.org/licenses/by/4.0/

(c) (i) Open Access

\section{Abstract}

In this paper, we derive and analyse rigorously a mathematical model of control strategies (screening, education, health care and immunization) of $\mathrm{HCV}$ in a community with inflow of infected immigrants. Both qualitative and quantitative analysis of the model is performed with respect to stability of the disease free and endemic equilibria. The results show that the disease free equilibrium is locally stable at threshold parameter less than unity and unstable at threshold parameter greater than unity. Using Lyapunov method, endemic equilibrium is globally stable under certain conditions. Numerical simulation of the model is implemented to investigate the sensitivity of certain key parameters on the HCV model in a community with inflow of infected immigrants. However, analysis shows that screening, education, health care and immunization have the effect of reducing the transmission of the disease in the community.

\section{Keywords}

HCV Disease, Infected Immigrants, Stability, Sensitivity Index, Lyapunov Method, Screening, Education, Health Care and Immunization

\section{Introduction}

Hepatitis C is a blood borne liver disease, caused by the Hepatitis C Virus (HCV), first identified by [1]. Moreover, the link between infectious diseases and screening must be understood in relation to infectives on the

\footnotetext{
"Corresponding author.
}

How to cite this paper: Ainea, N., Massawe, E.S., Makinde, O.D. and Namkinga, L. (2015) Mathematical Analysis of Control Strategies of HCV in a Community with Inflow of Infected Immigrants. Advances in Infectious Diseases, 5, 1-13. 
spread of HCV infections. [2] analysed the screening of HCV in a health maintenance Organization. Mathematical modelling of the spread of infectious diseases continues to become an important tool in understanding the dynamics of diseases and in decision making processes regarding diseases intervention programs for disease in many countries. For instance, [3] formulated and analysed a mathematical model on the effect of Treatment and Infected Immigrants on the spread of Hepatitis C Virus disease at Acute and Chronic stages. [4] considered SEI (Susceptible-Exposed-Infective) epidemic model with acute and chronic stages. [5] investigated the effects of a HCV educational intervention or a motivational intervention on alcohol use and sexual risk behaviours among injection drug users. [6] studied the potential impact of vaccination on the hep C virus epidemic in injection drug users. [7] presented the study on immunization strategies in chronic HCV infection. [8] reported that HCV patient education is associated with positive outcomes in various models of HCV care. However, in all the above studies, none of them incorporated the HCV infectiology and control strategies (screening, education, health care and immunization) in a community with inflow of infected immigrants. The aim of the paper is to have a deeper understanding of the effects of screening, education, health care and immunization in controlling the spread of HCV.

\section{Model Formulation}

A mathematical model is proposed and analysed to study the effect of screening, education, health care and immunization on the spread of HCV disease in the community. The model has five epidemiological classes: The susceptible $S$, exposed individuals $E$, the acute Infectives $A$, the chronic infectives $C$ and recovered group $R$. Total population at time $t$ is given by:

$$
N(t)=S(t)+E(t)+A(t)+C(t)+R(t)
$$

The interaction between the classes is being assumed as follows: Exposed individuals, acute infected and chronic infected immigrants enter into the population with the rates $\pi_{1}, \pi_{2}, \pi_{3}$ respectively. Susceptible individuals are infected with the HCV virus at a rate $v$, where $v=\frac{\beta_{1} A+\beta_{2} C}{N}$ and $\beta_{i}(i=1,2)$ are effective contact rate of individuals with acute and chronic hepatitis $\mathrm{C}$ respectively. It is assumed that the rate of contact of susceptibles with chronic individuals is much less than that of acute infectives $\left(\beta_{2} \leq \beta_{1}\right)$ because at chronic stage, people become aware of their infection and may choose to use control measures and change their behaviour and thus may contribute little in spreading the infection. The control variable based on screening programme aimed at, reduces the inflow of infected immigrants into the community at the rate $\tau$ and $\eta$ is the control variable based on education, health care and immunization to decrease the infection contact rate.

Taking into account the above considerations, we then have the following schematic flow diagram (Figure 1):

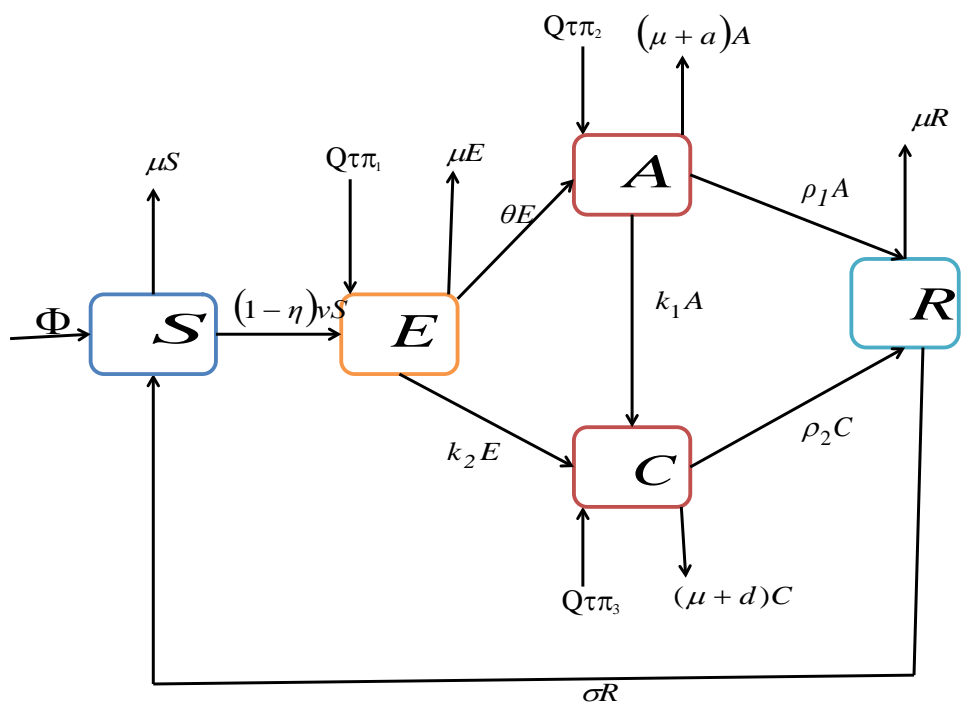

Figure 1. Model flowchart. 
From the above flow chart, and with

$$
v=\frac{\left(\beta_{1} A+\beta_{2} C\right)}{N} \text { and } \Phi=Q\left(1-\tau \pi_{1}-\tau \pi_{2}-\tau \pi_{3}\right),
$$

the model will be governed by the following system of equations:

$$
\begin{aligned}
\frac{\mathrm{d} S}{\mathrm{~d} t} & =Q\left(1-\tau \pi_{1}-\tau \pi_{2}-\tau \pi_{3}\right)-(1-\eta) v S+\sigma R-\mu S \\
\frac{\mathrm{d} E}{\mathrm{~d} t} & =Q \tau \pi_{1}+(1-\eta) v S-\left(\theta+k_{2}+\mu\right) E \\
\frac{\mathrm{d} A}{\mathrm{~d} t} & =Q \tau \pi_{2}+\theta E-\left(k_{1}+\rho_{1}+a+\mu\right) A \\
\frac{\mathrm{d} C}{\mathrm{~d} t} & =Q \tau \pi_{3}+k_{2} E+k_{1} A-\left(\rho_{2}+d+\mu\right) C \\
\frac{\mathrm{d} R}{\mathrm{~d} t} & =\rho_{1} A+\rho_{2} C-(\sigma+\mu) R
\end{aligned}
$$

with nonnegative initial conditions and $N(0)>0$.

where

$\beta_{i}(i=1,2)$ are the effective contact rates of individuals with acute and HCV respectively,

$\pi_{i}(i=1,2,3)$ are the rates at which exposed, acute and Chronic infected immigrants enter into the population respectively,

$Q$ is the recruitment rate,

$\theta$ is the rate of progression to acute infected class from exposed class,

$k_{i}(i=1,2)$ are the rates at which acute and exposed infective develop chronic respectively,

$\rho_{i}(i=1,2)$ are the rates at which acute and chronic individuals recovered respectively,

$a$ is the death rate of acute infected group due to the disease,

$\sigma$ is the rate at which infectious humans after recovery become immediately susceptible again,

$\mu$ is the natural death rate,

$d$ is the death rate of chronic infected group due to the disease,

$\tau$ is the screening rate of infected immigrants.

\section{Model Analysis}

The model system of Equations (2) will be analysed qualitatively to get insight into its dynamical features which will give a better understanding of the effects of screening, education, health care and immunization on the transmission of HCV infection in the population with inflow of infected immigrants. The threshold which governs elimination or persistence of HCV will be determined and studied. We begin by finding the invariant region and show that all solutions of system (2) are positive $\forall t>0$.

\subsection{Invariant Region}

In this section, a region in which solutions of the model system (2) are uniformly bounded is the proper subset

$$
\Omega \subset R_{+}^{5} \text {. }
$$

Let $(S, E, A, C, R) \in R_{+}^{5}$ be any solution with positive initial conditions. Then from Equation (2) it is noted that in the absence of the disease related mortality (i.e. $d=0, a=0$ ), the rate of change of the population is given by,

$$
\frac{\mathrm{d} N}{\mathrm{~d} t} \leq Q-\mu N
$$

Using Birkhoff and Rota's theorem [9] on the differential inequality (3), the following expression is obtained; 


$$
N \leq \frac{Q}{\mu}+\left(\frac{\mu N(0)-Q}{\mu}\right) \mathrm{e}^{-\mu t}
$$

where $N(0)$ is the value evaluated at the initial conditions of the respective variables.

Thus, as $t \rightarrow \infty$ in (4), the population size, $N \rightarrow \frac{Q}{\mu}$, which implies that $0 \leq N \leq \frac{Q}{\mu}$ In respectof this, all the feasible solutions of system (2) enter the region $\left\{\Omega=(S, E, A, C, R) \in R_{+}^{5}: N \leq \frac{Q}{\mu}\right\}$.

Hence, $\Omega$ is positively invariant and it issufficient to consider solutions in $\Omega$.

Furthermore, existence, uniqueness and continuation of results for system (2) hold in this region.

Lemma 1: The region $\Omega \subset R_{+}^{5}$ is positively invariant for the model system (2) with initial conditions in $R_{+}^{5}$.

\subsection{Positivity of Solutions}

Lemma 2: Let the initial data be $\left\{\{S(0), E(0), A(0), C(0), R(0) \geq 0\} \in R_{+}^{5}\right\}$ then, the solution set $\{S(t), E(t), A(t), C(t), R(t)\}$ of the system (2) is positive $\forall t>0$.

Proof:

From the first equation of the model system (2), we have

$$
\begin{gathered}
\frac{\mathrm{d} S}{\mathrm{~d} t}=Q\left(1-\tau \pi_{1}-\tau \pi_{2}-\tau \pi_{3}\right)-(1-\eta) \nu S+\sigma R-\mu S \\
\frac{\mathrm{d} S}{\mathrm{~d} t}+((1-\eta) v+\mu) S=Q\left(1-\tau \pi_{1}-\tau \pi_{2}-\tau \pi_{3}\right)+\sigma R .
\end{gathered}
$$

The Integration factor is $B(t)=\mathrm{e}^{\int_{0}^{t}((1-\eta) v(s)+\mu) \mathrm{d} s}$, multiplying both sides by the integration factor and integrating leads to

$$
\begin{gathered}
\frac{\mathrm{d}}{\mathrm{d} t}(S B(t))=B(t)\left[Q\left(1-\tau \pi_{1}-\tau \pi_{2}-\tau \pi_{3}\right)+\sigma R\right] \\
\Rightarrow B(t)=\mathrm{e}^{-\int_{0}^{t}((1-\eta) v(s)+\mu) \mathrm{ds}}\left(\int_{0}^{t}\left(Q\left(1-\tau \pi_{1}-\tau \pi_{2}-\tau \pi_{3}\right)+\sigma R\right) B(s) \mathrm{d} s+C\right) \geq 0 .
\end{gathered}
$$

Equations for $E(t), A(t), C(t)$ and $R(t)$ can be similarly be obtained.

Thus $S(t), E(t), A(t), C(t)$ and $R(t)$ are positive $\forall t \geq 0$.

\subsection{The Disease Free Equilibrium Point (DFE)}

In the absence of the disease, which implies that $\left(\pi_{1}=\pi_{2}=\pi_{3}=0, E=0, A=0, C=0, R=0\right)$, the disease free equilibrium points is given by

$$
\Delta_{01}=\left(S_{0}, E_{0}, A_{0}, C_{0}, R_{0}\right)=\left(\frac{Q}{\mu}, 0,0,0,0\right) .
$$

\subsection{The Effective Reproductive Number $\boldsymbol{R}_{e}$}

In this section, the threshold parameter that governs the spread of a disease which is called the effective reproduction number is determined. Mathematically, it is the spectral radius of the next generation matrix [10].

This definition is given for the models that represent spread of infection in a population. It is obtained by taking the largest (dominant) Eigen value, (spectral radius) of

$$
\left[\frac{\partial F_{i}\left(€_{0}\right)}{\partial X_{j}}\right] \cdot\left[\frac{\partial V_{i}\left(€_{0}\right)}{\partial X_{j}}\right]^{-1},
$$

where $F_{i}$ is the rate of appearance of new infection in compartment $i, V_{i}$ is the transfer of individuals out of 
the compartment $i$ by all other means and $\Delta_{0}$ is the disease free equilibrium.

Therefore,

$$
\boldsymbol{F}_{i}=\left(\begin{array}{c}
(1-\eta) \frac{\left(\beta_{1} A+\beta_{2} C\right)}{N} S \\
0 \\
0
\end{array}\right)
$$

and

$$
\boldsymbol{V}_{i}=\left(\begin{array}{c}
\left(\theta+k_{2}+\mu\right) E \\
\left(k_{1}+\rho_{1}+a+\mu\right) A-\theta E \\
\left(\rho_{2}+d+\mu\right) C-k_{2} E-k_{1} A
\end{array}\right) .
$$

The partial derivatives if (6) and (7) with respect to $E$, $A$ and $C$ gives

$$
\boldsymbol{F}=\left(\begin{array}{ccc}
0 & \frac{(1-\eta) \beta_{1} S}{N} & \frac{(1-\eta) \beta_{2} S}{N} \\
0 & 0 & 0 \\
0 & 0 & 0
\end{array}\right)
$$

and

$$
\boldsymbol{V}=\left(\begin{array}{ccc}
\theta+k_{2}+\mu & 0 & 0 \\
-\theta & k_{1}+\rho_{1}+a+\mu & 0 \\
-k_{2} & -k_{1} & \rho_{2}+d+\mu
\end{array}\right)
$$

In the absence of the disease and when $N=\frac{Q}{\mu}$, the matrix (8) becomes

$$
\boldsymbol{F}=\left(\begin{array}{ccc}
0 & (1-\eta) \beta_{1} & (1-\eta) \beta_{2} \\
0 & 0 & 0 \\
0 & 0 & 0
\end{array}\right)
$$

Now, taking the inverse of matrix (9) leads to

$$
\boldsymbol{V}^{-1}=\left(\begin{array}{ccc}
H 1 & H 2 & H 3 \\
0 & 0 & 0 \\
0 & 0 & 0
\end{array}\right)
$$

where

$$
\begin{aligned}
& H 1=\frac{(1-\eta) \beta_{1} \theta}{\left(\theta+k_{2}+\mu\right)\left(k_{1}+\rho_{1}+a+\mu\right)}+\frac{(1-\eta) \beta_{2}\left(\theta k_{1}+k_{1} k_{2}+k_{2} \rho_{1}+k_{2} a+k_{2} \mu\right)}{\left(\theta+k_{2}+\mu\right)\left(k_{1}+\rho_{1}+a+\mu\right)\left(\rho_{2}+d+\mu\right)} \\
& H 2=\frac{(1-\eta) \beta_{1}}{\left(k_{1}+\rho_{1}+a+\mu\right)}+\frac{(1-\eta) \beta_{2} k_{1}}{\left(k_{1}+\rho_{1}+a+\mu\right)\left(\rho_{2}+d+\mu\right)} \\
& H 3=\frac{(1-\eta) \beta_{2}}{\left(\rho_{2}+d+\mu_{C}\right)} .
\end{aligned}
$$

The spectral radius (dominant eigenvalue) of the matrix $\boldsymbol{F V}^{-1}$ is

$$
\frac{(1-\eta) \beta_{1} \theta}{\left(\theta+k_{2}+\mu\right)\left(k_{1}+\rho_{1}+a+\mu\right)}+\frac{(1-\eta) \beta_{2}\left(\theta k_{1}+k_{1} k_{2}+k_{2} \rho_{1}+k_{2} a+k_{2} \mu\right)}{\left(\theta+k_{2}+\mu\right)\left(k_{1}+\rho_{1}+a+\mu\right)\left(\rho_{2}+d+\mu\right)}
$$


Hence, the effective reproduction number of the model system (2) is given by

$$
R_{e}=\frac{(1-\eta) \beta_{1} \theta}{\left(\theta+k_{2}+\mu\right)\left(k_{1}+\rho_{1}+a+\mu\right)}+\frac{(1-\eta) \beta_{2}\left(\theta k_{1}+k_{1} k_{2}+k_{2} \rho_{1}+k_{2} a+k_{2} \mu\right)}{\left(\theta+k_{2}+\mu\right)\left(k_{1}+\rho_{1}+a+\mu\right)\left(\rho_{2}+d+\mu\right)} .
$$

The effective reproduction number $R_{e}$ measures the average number of new infections generated by a typical infectious individual in a community with inflow of infected immigrants when screening, education, health care and immunization strategies are in place.

Theorem 1: The disease free equilibrium of the model system (2) is locally asymptotically stable if $R_{e}<1$ and unstable if $R_{e}>1$.

Theorem 1 implies that HCV can be eliminated from the community when $R_{e}<1$ if the initial size of the sub population of the model are in the basin of attraction of the disease free equilibrium. That means if $R_{e}<1$, then on average an infected individual produce less than one new infected individual over the course of its infectious period and the infection cannot grow.

From Equation (12), for $R_{e}$ to be less than 1 this will only be possible when $\eta$ (which implies HCV education, health care and immunization) are increased without bound in collaboration with other intervention strategies to all people including immigrants in a given locality which may result into the decreasing effect on $\beta_{1}, \beta_{2}$.

In the absence of interventions (screening, education, health care and immunization) that is $(\tau=0, \eta=0)$, $R_{e}$ is reduced to:

$$
R_{0}=\frac{\beta_{1} \theta}{\left(\theta+k_{2}+\mu\right)\left(k_{1}+\rho_{1}+a+\mu\right)}+\frac{\beta_{2}\left(\theta k_{1}+k_{1} k_{2}+k_{2} \rho_{1}+k_{2} a+k_{2} \mu\right)}{\left(\theta+k_{2}+\mu\right)\left(k_{1}+\rho_{1}+a+\mu\right)\left(\rho_{2}+d+\mu\right)} .
$$

Thus $R_{e}<R_{0}$. Hence the presence of screening, education, health care and immunization can eradicate the $\mathrm{HCV}$ infection if $R_{e}$ can be reduced to below unity.

\subsection{Local Stability of Disease Free Equilibrium (DFE)}

Local stability of disease free equilibrium $\Delta_{01}$, can be determined by the variational matrix $\boldsymbol{J}_{01}$ of the model system (2). The Jacobian matrix at the steady states is given by;

$$
\boldsymbol{J}_{01}=\left[\begin{array}{ccccc}
-\mu & 0 & -\beta_{1}(1-\eta) & -\beta_{2}(1-\eta) & 0 \\
0 & -\left(\theta+k_{2}+\mu\right) & \beta_{1}(1-\eta) & \beta_{2}(1-\eta) & 0 \\
0 & \theta & -\left(k_{1}+\rho_{1}+a+\mu\right) & 0 & 0 \\
0 & k_{2} & k_{1} & -\left(\rho_{2}+d+\mu\right) & 0 \\
0 & 0 & \left(\rho_{1+a}\right) & \rho_{2} & -(\delta+\mu)
\end{array}\right]
$$

The local stability analysis of the Jacobian matrix (13) of the system (2) can be done by the trace/determinant method. Where by matrix $\left(\boldsymbol{J}_{01}\right)$ is locally asymptotically stable if and only if the trace of matrix $\left(\boldsymbol{J}_{01}\right)$ is strictly negative and its determinant is strictly positive. Whose trace and determinant are given by

$$
\operatorname{Trace}\left(\boldsymbol{J}_{01}\right)=-\mu-\left(\theta+k_{2}+\mu\right)-\left(k_{1}+\rho_{1}+a+\mu\right)-\left(\rho_{2}+d+\mu\right)-(\delta+\mu)<0
$$

and

$$
\operatorname{det}\left(\boldsymbol{J}_{01}\right)=r_{1} r_{2} r_{3} r_{4}-\theta p_{1} r_{3} r_{4}-\theta k_{1} p_{2} r_{4}-k_{2} r_{2} p_{2} r_{4}
$$

where $r_{1}=\theta+k_{2}+\mu, r_{2}=k_{1}+\rho_{1}+a+\mu, \quad r_{3}=\rho_{2}+d+\mu, \quad r_{4}=\sigma+\mu, \quad p_{1}=(1-\eta) \beta_{1}, \quad p_{2}=(1-\eta) \beta_{2}$.

Hence $\operatorname{det}\left(\boldsymbol{J}_{01}\right)>0$ if $\theta p_{1} r_{3} r_{4}+\theta k_{1} p_{2} r_{4}+k_{2} r_{2} p_{2} r_{4}<r_{1} r_{2} r_{3} r_{4}$.

That is equivalent to

$$
\frac{\left(\theta p_{1} r_{3} r_{4}+\theta k_{1} p_{2} r_{4}+k_{2} r_{2} p_{2} r_{4}\right)}{r_{1} r_{2} r_{3} r_{4}}<1
$$

since $\frac{\left(\theta p_{1} r_{3} r_{4}+\theta k_{1} p_{2} r_{4}+k_{2} r_{2} p_{2} r_{4}\right)}{r_{1} r_{2} r_{3} r_{4}}=R_{e}$.

Thus, $\Delta_{01}$ is locally asymptotically stable if and only if $R_{e}<1$. These results are summarized with the 
theorem 1.

\subsection{The Endemic Equilibrium Point $\Delta$}

Endemic equilibrium point $\Delta$ is a steady state solution in which the disease persists in the population (i.e. $E \neq 0, A \neq 0, C \neq 0, R \neq 0$ )

$$
\begin{aligned}
& S^{*}=\frac{R_{e} A^{*}-r_{17}}{\left(r_{10}+r_{13} R_{e}-r_{14}\right) A^{*}+r_{11}} \\
& E^{*}=\frac{\left(k_{1}+\rho_{1}+a+\mu\right) A^{*}-\tau Q \pi_{2}}{\theta} \\
& C^{*}=r_{2}+\left(\frac{R_{e} r_{1}-(1-\eta) \beta_{1} \theta}{(1-\eta) \beta_{2} \theta}\right) A^{*} \\
& R^{*}=\frac{\left[r_{5}+\rho_{2}\left(R_{e} r_{1}-r_{3}\right)\right]}{r_{7}} A^{*}+r_{6}
\end{aligned}
$$

where,

$$
\begin{gathered}
r_{1}=\left(\theta+k_{2}+\mu\right)\left(k_{1}+\rho_{1}+a+\mu\right), \quad r_{2}=\theta \tau Q \pi_{3}-k_{2} \tau Q \pi_{3}, \quad r_{3}=(1-\eta) \beta_{1} \theta, \\
r_{4}=(1-\eta) \beta_{2} \theta, \quad r_{5}=\rho_{1} r_{4}, \quad r_{6}=\rho_{2} r_{2} r_{4}, \quad r_{7}=r_{4}(\sigma+\mu), \quad r_{8}=\frac{\theta+k_{2}+\mu}{\theta}, \\
r_{9}=k_{1}+\rho_{1}+a+\mu, \quad r_{10}=\frac{(1-\eta) \beta_{1}}{N}, \quad r_{11}=\frac{(1-\eta) \beta_{2} r_{2}}{N}, \quad r_{12}=\frac{(1-\eta) \beta_{2}}{r_{5} N}, \\
r_{13}=r_{1} r_{12}, \quad r_{14}=r_{3} r_{12}, \quad r_{15}=r_{8} Q \tau \pi_{2}, \quad r_{16}=r_{8} r_{9}, \quad r_{17}=Q \tau \pi_{1}+r_{15}, \\
r_{18}=Q\left(1-\tau \pi_{1}-\tau \pi_{2}-\tau \pi_{3}\right), \quad r_{19}=r_{18} r_{10} r_{7}-r_{18} r_{14} r_{7}, \quad r_{20}=r_{18} r_{13} r_{7}, \quad r_{21}=r_{18} r_{11} r_{7}
\end{gathered}
$$

and $A^{*}$ is the solution of the quadratic polynomial

$$
f\left(A^{*}\right)=G\left(A^{*}\right)^{2}+H\left(A^{*}\right)+K=0
$$

where,

$$
\begin{aligned}
& G= {\left[\sigma r_{5}+\sigma \rho_{2}\left(r_{1} R_{e}-r_{3}\right)\right]\left[r_{10}+r_{13} R_{e}-r_{4}\right]+r_{7} r_{16}\left(r_{10}+r_{13} R_{e}-r_{14}\right) } \\
& H=\left(r_{19}+r_{20} R_{e}\right)+r_{11} r_{7} r_{16}-r_{7} r_{17}\left(r_{10}+r_{13} R_{e}-r_{14}\right)+r_{11}\left[\sigma r_{5}+\sigma \rho_{2}\left(r_{1} R_{e}-r_{3}\right) r\right] \\
&+\sigma r_{6}\left(r_{10}+r_{13} R_{e}-r_{14}\right)-r_{16} \mu \\
& K=r_{21}-r_{11} r_{7} r_{17}+\sigma r_{6} r_{11}+r_{17} r_{7} \mu .
\end{aligned}
$$

The equation, $f\left(A^{*}\right)=0$ corresponds to a situation when the disease persists (endemic). In case of backward bifurcation, multiple endemic equilibrium must exist.

However it is important to note that $K$ is always positive if $R_{e}<1$ and negative if $R_{e}>1$.

Theorem 2: The HCV model with screening, education, health care and immunization interventions have:

i) Precisely one unique endemic equilibrium if $K<0 \Leftrightarrow R_{e}>1$.

ii) Precisely one unique endemic equilibrium if $H<0$ and $K=0$ or $H^{2}-4 G K=0$.

iii) Precisely two endemic equilibrium if $K>0, H<0$ and $H^{2}-4 G K>0$.

iv) None otherwise.

Theorem 3: A unique endemic equilibrium point, $\Delta$ exists if and only if $R_{e}>1$.

\subsection{Global Stability of the Endemic Equilibrium Point $\Delta$}

The global stability of the endemic equilibrium $\Delta$ is analysed using the following constructed Lyapunov function by [11] 
Theorem 4: If $R_{e}>1$, the endemic equilibrium $\Delta$ of the model (2) is globally asymptotically stable.

Proof: To establish the global stability of the endemic equilibrium $\Delta$, we construct the following Lyapunov function:

$$
\begin{aligned}
& V\left(S^{*}, E^{*}, A^{*}, C^{*}, R^{*}\right)=\left(S-S^{*}-S^{*} \log \frac{s^{*}}{S}\right)+\left(E-E^{*}-E^{*} \log \frac{E^{*}}{E}\right) \\
& +\left(A-A^{*}-A^{*} \log \frac{A^{*}}{A}\right)+\left(C-C^{*}-C^{*} \log \frac{C^{*}}{C}\right)+\left(R-R^{*}-R^{*} \log \frac{R^{*}}{R}\right) .
\end{aligned}
$$

By direct calculating the derivative of $V$ along the solution of (2) we have;

$$
\frac{\mathrm{d} V}{\mathrm{~d} t}=\left(\frac{S-S^{*}}{S}\right) \frac{\mathrm{d} S}{\mathrm{~d} t}+\left(\frac{E-E^{*}}{E}\right) \frac{\mathrm{d} E}{\mathrm{~d} t}+\left(\frac{A-A^{*}}{A}\right) \frac{\mathrm{d} A}{\mathrm{~d} t}+\left(\frac{C-C^{*}}{C}\right) \frac{\mathrm{d} C}{\mathrm{~d} t}+\left(\frac{R-R^{*}}{R}\right) \frac{\mathrm{d} R}{\mathrm{~d} t}
$$

or

$$
\frac{\mathrm{d} V}{\mathrm{~d} t}=P-Q
$$

where,

$$
\begin{aligned}
P= & \left(\frac{\left(S-S^{*}\right)^{2}}{S}\right)\left[\left(\frac{(1-\eta)\left(\beta_{1} A+\beta_{2} C\right)}{N}\right)-\mu\right]+\sigma R+\frac{S^{*}}{S} \delta R^{*}+\left(\frac{(1-\eta)\left(\beta_{1} A+\beta_{2} C\right)}{N}\right) S \\
& +\left(\frac{(1-\eta)\left(\beta_{1} A^{*}+\beta_{2} C^{*}\right)}{N}\right) S^{*}+\theta E+\frac{A^{*}}{A} \theta E^{*}+k_{2} E+k_{1} A+\frac{C^{*}}{C} k_{2} E^{*}+\frac{C^{*}}{C} k_{1} A^{*} \\
& +\rho_{1} A+\rho_{2} C+\frac{R^{*}}{R} \rho_{1} A^{*}+\frac{R^{*}}{R} \rho_{2} C^{*}+Q\left(1-\tau \pi_{1}-\tau \pi_{2}-\tau \pi_{3}\right)+\tau \pi_{1} Q+\tau \pi_{2} Q+\tau \pi_{3} Q \\
Q= & -\delta R^{*}-\frac{S^{*}}{S} \delta R-\left(\frac{\left(E-E^{*}\right)^{2}}{E}\right)\left[\theta+k_{2}+\mu\right]-\left(\frac{(1-\eta)\left(\beta_{1} A+\beta_{2} C\right)}{N}\right) S^{*} \\
& -\frac{S^{*}}{S} Q\left(1-\tau \pi_{1}-\tau \pi_{2}-\tau \pi_{3}\right)-\left(\frac{(1-\eta)\left(\beta_{1} A^{*}+\beta_{2} C^{*}\right)}{N}\right) S \\
& -\left(\frac{\left(A-A^{*}\right)^{2}}{A}\right)\left[k_{1}+\rho_{1}+a+\mu\right]-\theta E^{*}-\frac{A^{*}}{A} \theta E-\frac{A^{*}}{A} \pi_{2} Q-\frac{E^{*}}{E} \tau \pi_{1} Q-\frac{C^{*}}{C} \tau \pi_{3} Q \\
& -\left(\frac{\left(C-C^{*}\right)^{2}}{C}\right)\left[\rho_{2}+d+\mu\right]-k_{2} E-k_{1} A^{*}-\frac{C^{*}}{C} k_{2} E-\frac{C^{*}}{C} k_{1} A \\
& -\left(\frac{\left(R-R^{*}\right)^{2}}{R}\right)[\delta+\mu]-\rho_{1} A^{*}-\rho_{2} C^{*}-\frac{R^{*}}{R} \rho_{1} A-\frac{R^{*}}{R} \rho_{2} C .
\end{aligned}
$$

Thus if $P<Q$ then $\frac{\mathrm{d} V}{\mathrm{~d} t} \leq 0$; Noting that $\frac{\mathrm{d} V}{\mathrm{~d} t}=0$ if and only if $S=S^{*} ; E=E^{*} ; A=A^{*} ; C=C^{*}$; $R=R^{*}$ : Therefore, the largest compact invariant set in $\left\{\left(S^{*}, E^{*}, A^{*}, C^{*}, R^{*}\right) \in \Omega: \frac{\mathrm{d} V}{\mathrm{~d} t}=0\right\}$ is the singleton $\{\Delta\}$ where $\Delta$ is the endemic equilibrium of the system (2). By LaSalle's invariant principle, it implies that $\Delta$ 
is globally asymptotically stable in $\Omega$ if $P<Q$.

\subsection{Numerical Sensitivity Analysis}

In determining how best to reduce human mortality and morbidity due to HCV, we calculate the sensitivity indices of the basic reproduction number, $R_{e}$ to the parameters in the model using approach of [12]. These indices are crucial in determining the importance of each individual parameter in transmission dynamics and prevalence of the disease. Sensitivity analysis determines parameters that have a high impact on $R_{e}$ and should be targeted by intervention strategies. Sensitivity indices allow us to measure the relative change in a state variablewhen a parameter changes [12]. When a variable is a differentiable function of the parameter, the sensitivity index may be alternatively defined using partial derivatives.

Numerical values of sensitivity indices of $R_{e}$ to parameter values for the HCV model, evaluated using the following estimated parameter values: $\beta_{1}=0.35, \beta_{2}=0.2, \theta=0.5, k_{1}=0.3, k_{2}=0.4, \rho_{1}=0.2$, $\rho_{2}=0.25, a=0.5, d=0.05, \eta=0.4$ and $\mu=0.02$.

Definition 1: The normalised forward sensitivity index of a variable " $p$ " that depends differentiable on a parameter " $q$ " is defined as:

$$
X_{q}^{p}=\frac{\partial p}{\partial q} \times \frac{q}{p} .
$$

Having an explicit formula for $R_{e}$ in Equation (18), we derive an analytical expression for the sensitivity of $R_{e}$ as $X_{q}^{R_{e}}=\frac{\partial R_{e}}{\partial q} \times \frac{q}{R_{e}}$ to each of parameters involved in $R_{e}$. For example the sensitivity indices of $R_{e}$ with respect to $\beta_{1}$ and $\theta$ are given by;

$$
X_{\beta_{1}}^{R_{e}}=\frac{\partial R_{e}}{\partial \beta_{1}} \times \frac{\beta_{1}}{R_{e}}=0.3341288785 \text { and } X_{\theta}^{R_{e}}=\frac{\partial R_{e}}{\partial \theta} \times \frac{\theta}{R_{e}}=-0.0303517690 .
$$

Other indices

$$
X_{\beta_{3}}^{R_{e}}, X_{\eta}^{R_{e}}, X_{a}^{R_{e}}, X_{\rho_{2}}^{R_{e}}, X_{\rho_{1}}^{R_{e}}, X_{d}^{R_{e}}, X_{\mu}^{R_{e}}, X_{k_{1}}^{R_{e}} \text { and } X_{k_{2}}^{R_{e}},
$$

are obtained following the same method and tabulated as follows:

From Table 1, it can be observed that when the parameters $\beta_{2}, \beta_{1}, k_{2}$ and $k_{1}$ are increased keeping the other parameters constant they increase the value of $R_{e}$ implying that they increase the endemicity of the disease as they have positive indices. While the parameters $\eta, a, \rho_{2}, \rho_{1}, d, \theta$ and $\mu$ decrease the value of $R_{e}$ when they are increased while keeping the other parameters constant, implying that they decrease the

Table 1. Numerical values of sensitivity indices of $R_{e}$.

\begin{tabular}{cc}
\hline Parameter Symbol & Sensitivity Index \\
\hline$\beta_{2}$ & -0.666666666 \\
$\rho_{2}$ & 0.6658711218 \\
$\beta_{1}$ & -0.520211814 \\
$a$ & 0.3341288785 \\
$d$ & -0.251532594 \\
$\rho_{1}$ & -0.104042362 \\
$\mu$ & -0.100613037 \\
$k_{2}$ & -0.0734173793 \\
$\theta$ & 0.05209089976 \\
$k_{1}$ & -0.0303517690 \\
\hline
\end{tabular}


endemicity of the disease as they have negative indices.

The specific interpretation of each parameter shows that, the most sensitive parameter is the control based on education, health care and immunization $\eta$, followed by effective contact rate of individuals with chronic disease $\beta_{2}$, then recovered rate of chronic individuals due to treatment $\rho_{2}$, effective contact rate of individuals with acute $\beta_{1}$, followed by recovery rate naturally from acute $a$, death rate of chronic infected $d$, recovered rate of acute individuals due to treatment $\rho_{1}$, natural mortality rate $\mu$, rate at which exposed develop chronic $k_{2}$, the rate at which acute infective are detected by a screening method from exposed group $\theta$, the rate at which screened develop to chronic $k_{1}$, which is the least sensitive parameter.

\subsection{Numerical Simulations}

In this section, we illustrate the analytical results of the study by carrying out numerical simulations of the model system (2) using the following estimated parameter values: $\beta_{1}=0.38, \beta_{2}=0.001, \theta=0.5, k_{1}=0.5, k_{2}=0.34$, $\rho_{1}=[0.02-0.5], \rho_{2}=[0.002-0.45], \sigma=[0.13-0.5], a=0.0034, d=0.5, \quad \mu=[0.0001-0.09]$, $Q=[5-85], \pi_{1}=[0.4-0.6], \pi_{2}=0.2, \pi_{3}=0.03, N=[2-15]$.

Figures 2(a)-(d) show the proportion of HCV exposed, infective populations (acute, chronic) and proportion of HCV infectives all plotted against the proportion of susceptible population. This shows the dynamic beha-

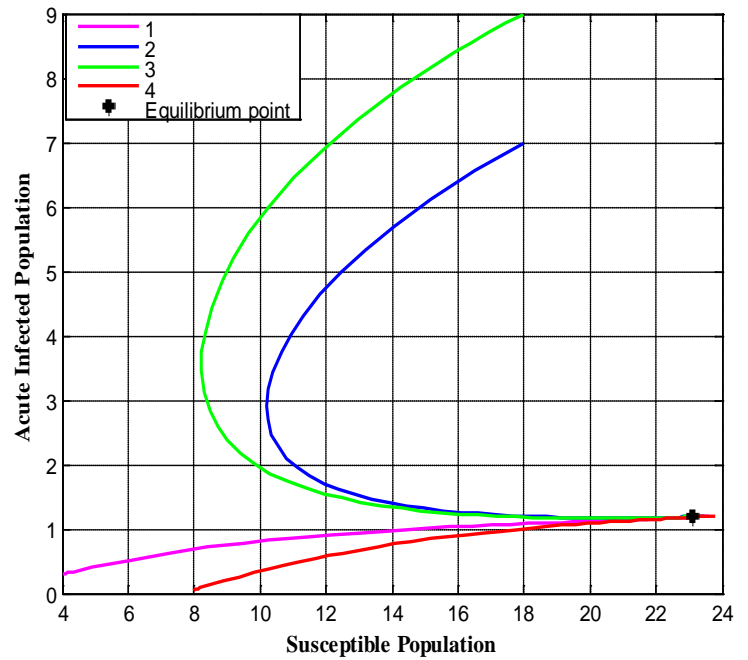

(a)

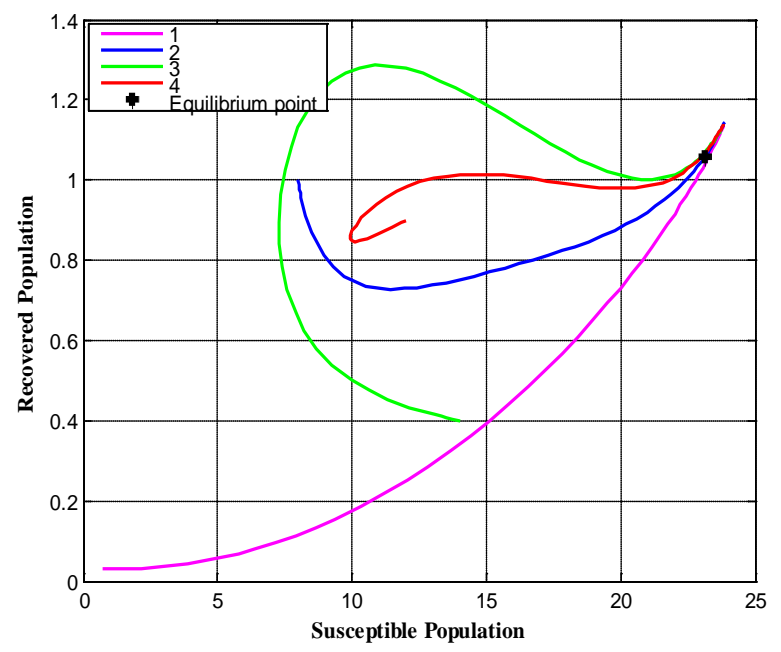

(c)

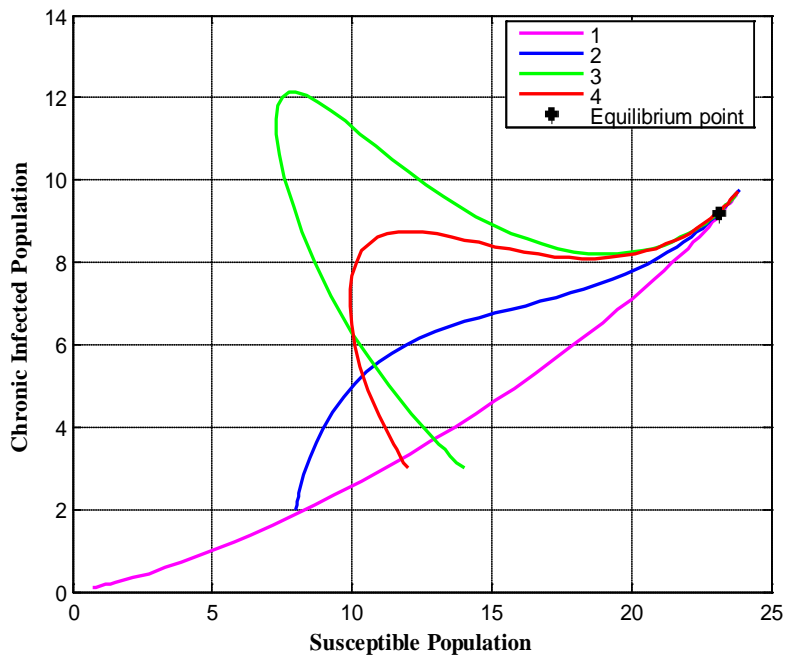

(b)

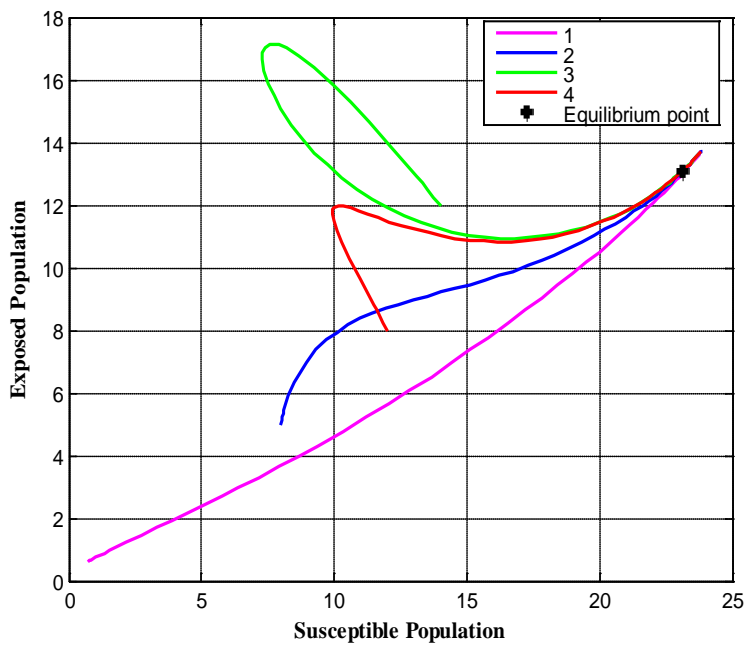

(d)

Figure 2. Phase portrait of the dynamics of susceptibles and the infected and recovered population. 
viour of the endemic equilibrium of the model system (2) using the estimated parameter values above.

The phase portrait in Figures 2(a)-(d) shows that for any initial starting point or initial value, the solution curves tend to the endemic equilibrium point $\Delta$. Hence, we infer that the system (2) is globally stable about the endemic equilibrium point $\Delta$ for the set of parameters above.

In Figures 3(a)-(d), the variation of proportions of exposed, recovered, acute and chronic infective populations for different rates of education, health care and immunization $(\eta)$ is shown.

Figures 3(a)-(d), shows that the infected population decreases as the control strategies (education, health care and immunization), $\eta$ increased. This confirms that, if $\eta$ is not effective the disease will invade the population.

Figures 4(a)-(d) shows the variation of proportions of exposed, acute and chronic infective populations and recovered population for different rates of screening.

From Figures 4(a)-(d) we vary the screened rate of infected immigrants, and it is seen that as the degree of screening increases, the infected population decreases. The results further show that increasing the screening rate, decreases the severity of the epidemic. Once again this confirms that, screening can reduce the inflow of infected immigrants into the community.

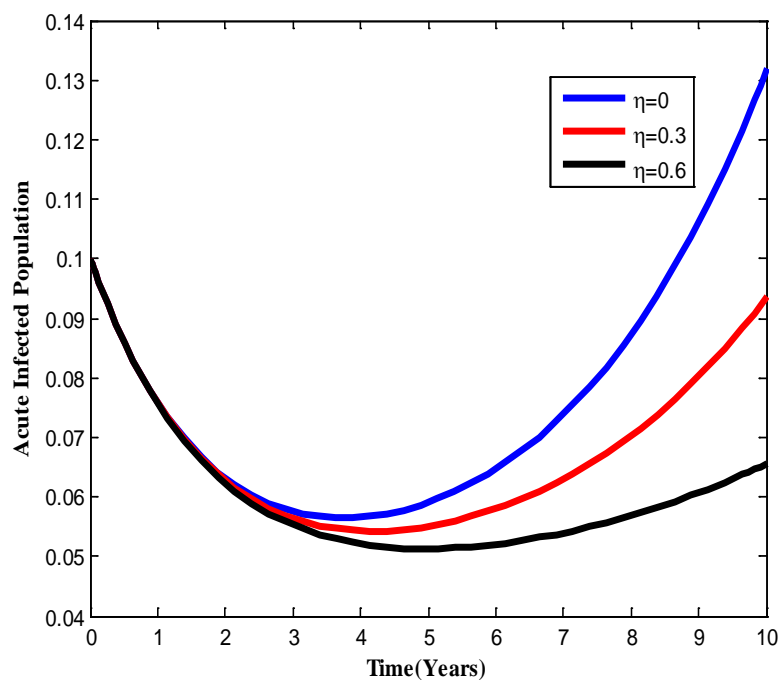

(a)

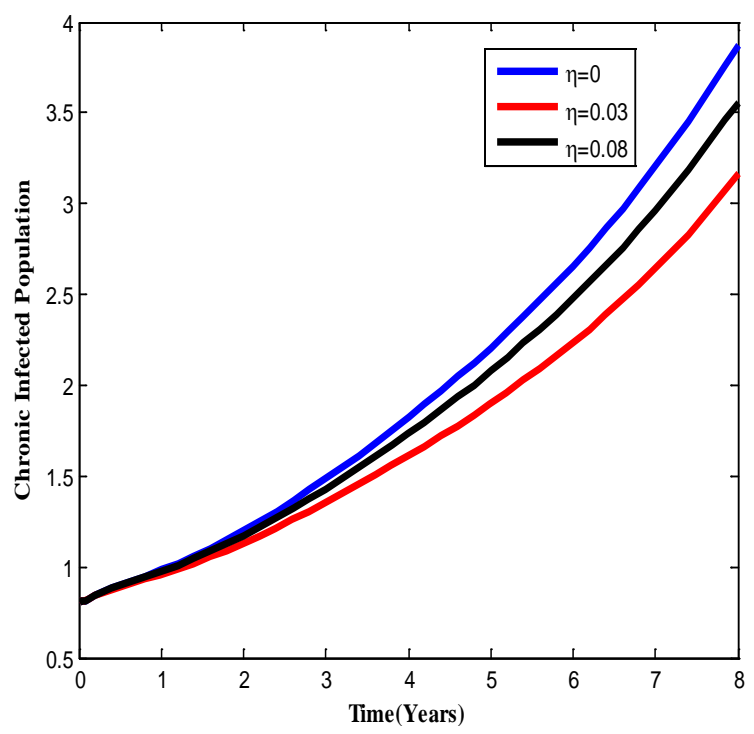

(c)

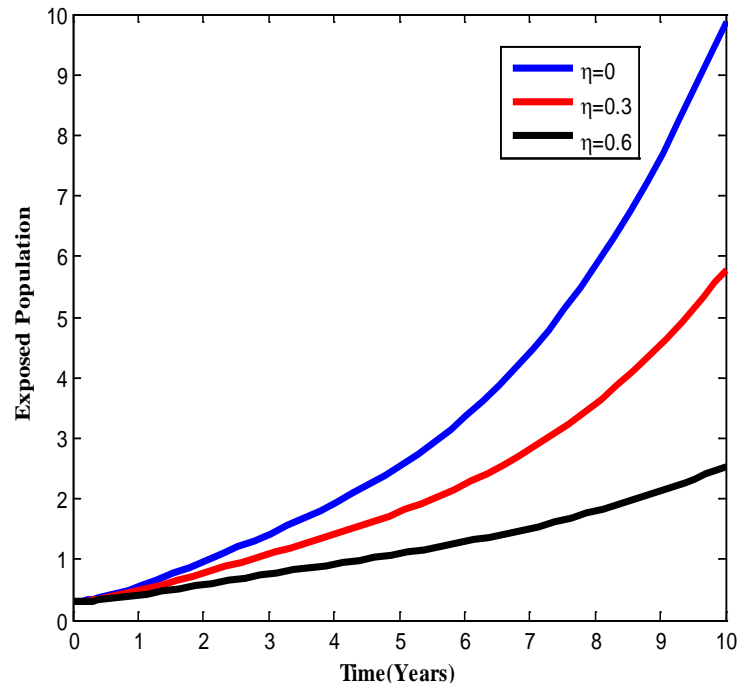

(b)

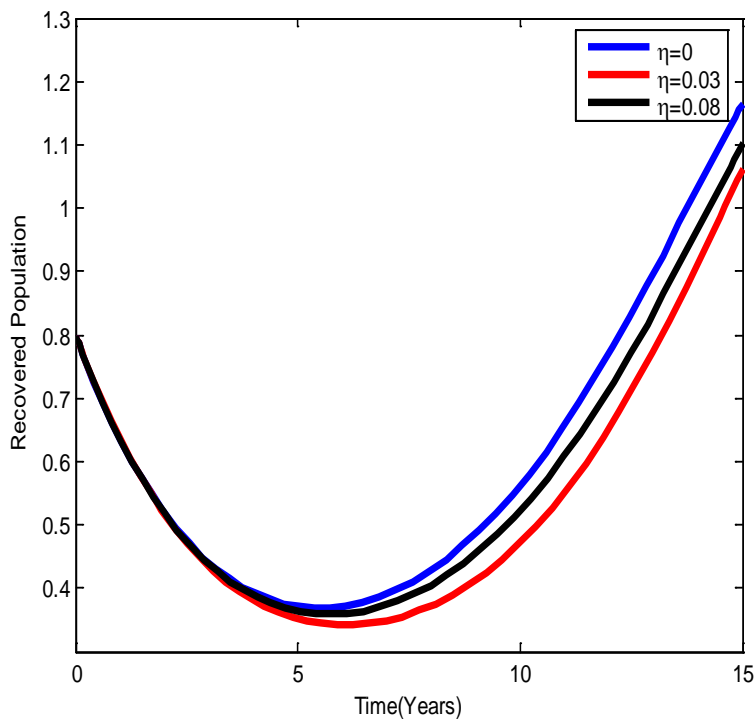

(d)

Figure 3. Variation population under different values of $\eta$ (education, health care and immunization). 


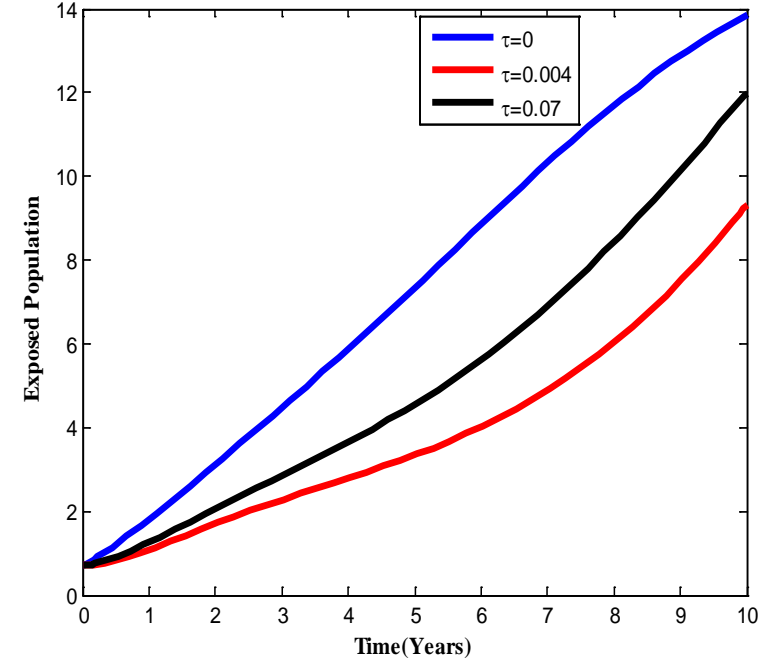

(a)

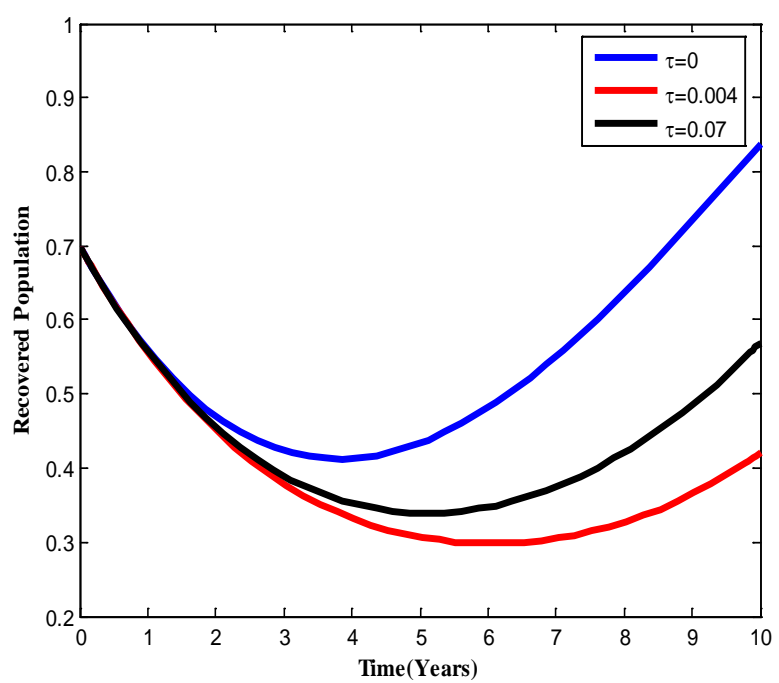

(c)

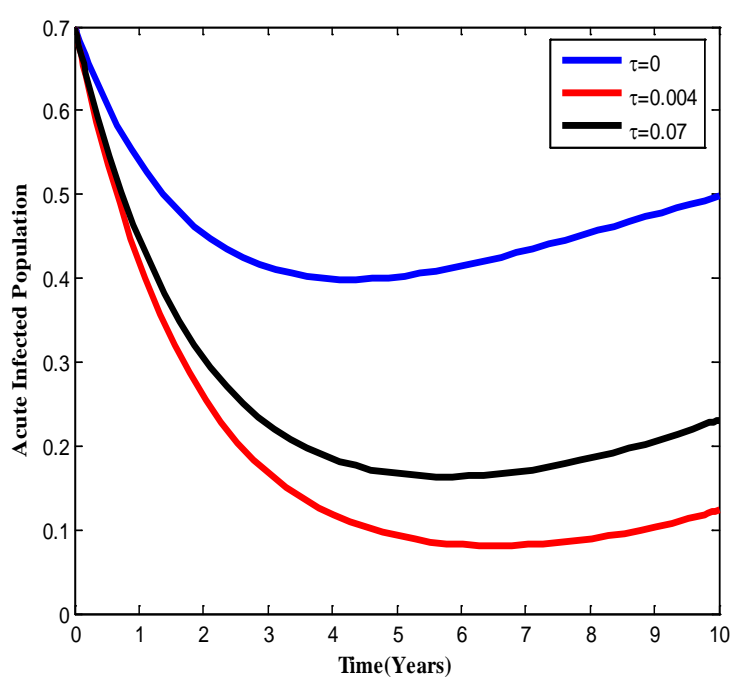

(b)

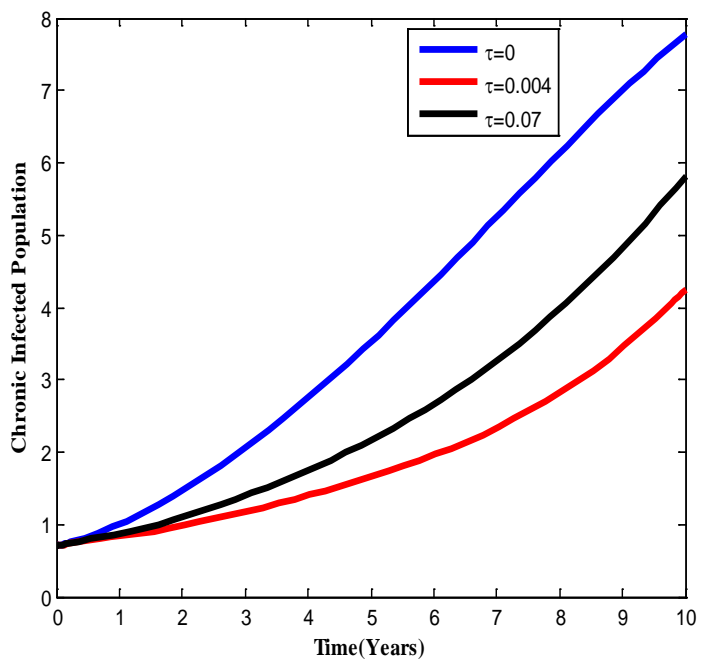

(d)

Figure 4. Variation of population under different values of $\tau$ (screening).

\section{Discussions and Conclusion}

In this paper, a mathematical model of control strategies of HCV in a community with inflow of infected immigrants been established. Both qualitative and numerical analysis of the model was done. The model incorporates the assumption that infected immigrants enter in the community. It is shown that there exists a feasible region where the model is well posed in which a unique disease free equilibrium point exists. The disease free and endemic equilibrium points were obtained and their stabilities investigated. The model showed that the disease free equilibrium is locally stable at threshold parameter less than unity and unstable at threshold parameter greater than unity. Using Lyapunov method, endemic equilibrium is globally stable under certain conditions. A sensitivity analysis shows that the control based on education, health care and immunization $\eta$ is the most sensitive parameter on $R_{e}$ and the least is $k_{1}$. A numerical study of the model has been conducted to see the effect of certain key parameters on the spread of the disease. It was observed that the spread of the disease decreases due to the presence of control strategies (screening, education, health care and immunization). As the control strategies increase, the exposed, acute and chronic infective individuals also decrease in the population. Finally, from the analysis, it may be hypothesized that preventive measures, through reducing rates of transmission of HCV are therefore necessary to the community. Since reduced transmission leads to lower prevalence of the disease in 
the long-term, the national health care to HCV should therefore seek to ensure that all people at risk or that have been at risk in the past, have access to and are supported in the use of HCV screening, education, health care immunization, regardless of their social and economic status.

\section{References}

[1] Choo, L., Kuo, G. and Weiner, A.J. (1989) Isolation of a cDNA Clone Derived from a Blood-Borne Non-A, Non-B Viral Hep Atitis Genome. PubMed, 244, 359-362.

[2] Fischer, L.R., Tope, D.H., Kathleen, S., Conboy, R.N., Hedblom, B.D., Ronberg, E., Shewmake, D.K. and Butter, J.C. (2000) Screening for Hepatitis C Virus in a Health Maintenance Organisation, American Medical Association.

[3] Ainea, N., Massawe, E.S. and Makinde, O.D. (2012) Modelling the Effect of Treatment and Infected Immigrants on the Spread of Hepatitis C Virus Disease with Acute and Chronic Stages. American Journal of Computational and Applied Mathematics, 1, 10-20. http://dx.doi.org/10.5923/j.ajcam.20120201.02

[4] Cai, L. and Li, X. (2008) A Note on Global Stability of an SEI Epidemic Model with Acute and Chronic Stages. Applied Maths and Computations, 196, 923-930. http://dx.doi.org/10.1016/j.amc.2007.07.024

[5] Zule, W.A., Costenbader, E.C, Coomes, C.M. and Wechsberg, W.M. (2009) Effects of a Hepatitis C Virus Educational in Tervention or a Motivational Intervention on Alcohol Use, Injection Drug Use, and Sexual Risk Behaviours among Injection Drug Users. American Journal of Public Health, 1, 180-186. http://dx.doi.org/10.2105/AJPH.2007.126854

[6] Hahn, J.A., Wylie, D., Dill, J., Sanchez, M.S., Lioyd-Smith, J.O., Pageshefer, K. and Getz, W.M. (2009) Potential Impact on Vaccination on the Hepatitis C Virus Epidemic in Injection Drug Users. PubMed, 1, 47-57.

[7] Encke, J., Findeklee, J., Geib, J., Pfaff, E. and Stremmel, W. (2005) Prophylactic and Therapeutic Vaccination with Dendritic Cells against Hepatitis C Virus Infection. Clinical and Experimental Immunology, 142, 362-369. http://dx.doi.org/10.1111/j.1365-2249.2005.02919.x

[8] Lubega, S., Agbim, U., Surjadi, M., Mahoney, M. and Khalili, M. (2013) Formal Hepatitis C Education Enhances HCV Care Coordination, Expedites HCV Treatment and Improves Antiviral Response Liver International. Liver International, 33, 999-1007. http://dx.doi.org/10.1111/liv.12150

[9] Michael, J. (2011) Mathematical Analysis of the Effect of Screening and Vaccination on the Dynamics of HIV/AIDS. MSc. Dissertation, University of Dar es Salaam, Dar es Salaam.

[10] Van den Driessche, P. and Watmough, J. (2002) Reproduction Numbers and Sub-Threshold Endemic Equilibria for Compartmental Models of Disease Transmission. Mathematical Bio-Sciences, 180, 29-48. http://dx.doi.org/10.1016/S0025-5564(02)00108-6

[11] Cai, L. and Li. Z. (2010) Analysis of a Simple Vector-Host Epidemic Model with Direct Transmissionl. College of Mathematics and Information Science, China.

[12] Chitnis, N., Hyman, J.M. and Cushing, J.M. (2008) Determining Important Parameters in the Spread of Malaria through the Sensitivity Analysis of a Mathematical Model. Bulletin of Mathematical Biology, 70, 1272-1296. http://dx.doi.org/10.1007/s11538-008-9299-0 
Scientific Research Publishing (SCIRP) is one of the largest Open Access journal publishers. It is currently publishing more than 200 open access, online, peer-reviewed journals covering a wide range of academic disciplines. SCIRP serves the worldwide academic communities and contributes to the progress and application of science with its publication.

Other selected journals from SCIRP are listed as below. Submit your manuscript to us via either submit@scirp.org or Online Submission Portal.
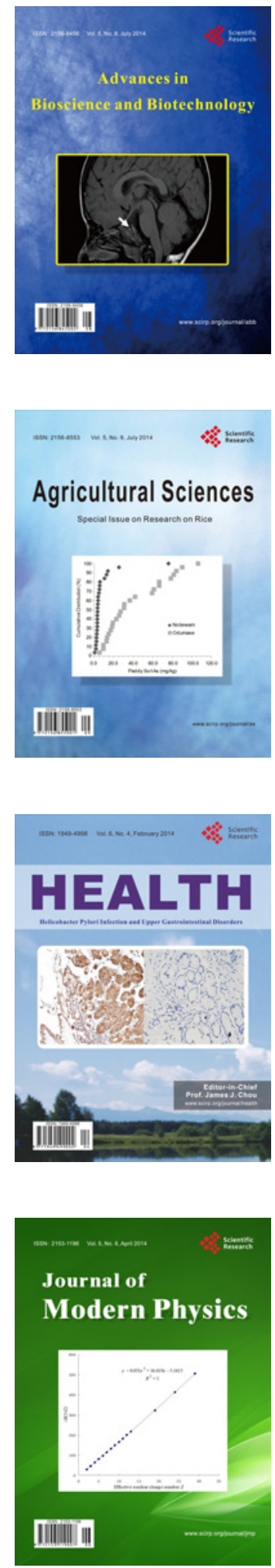
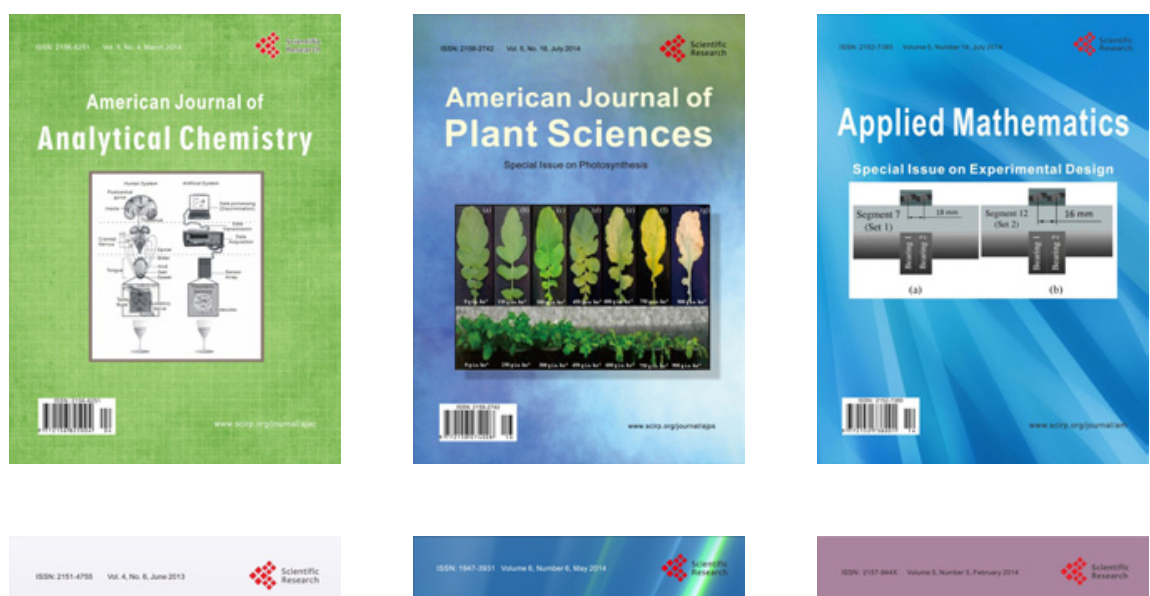

Creative Education
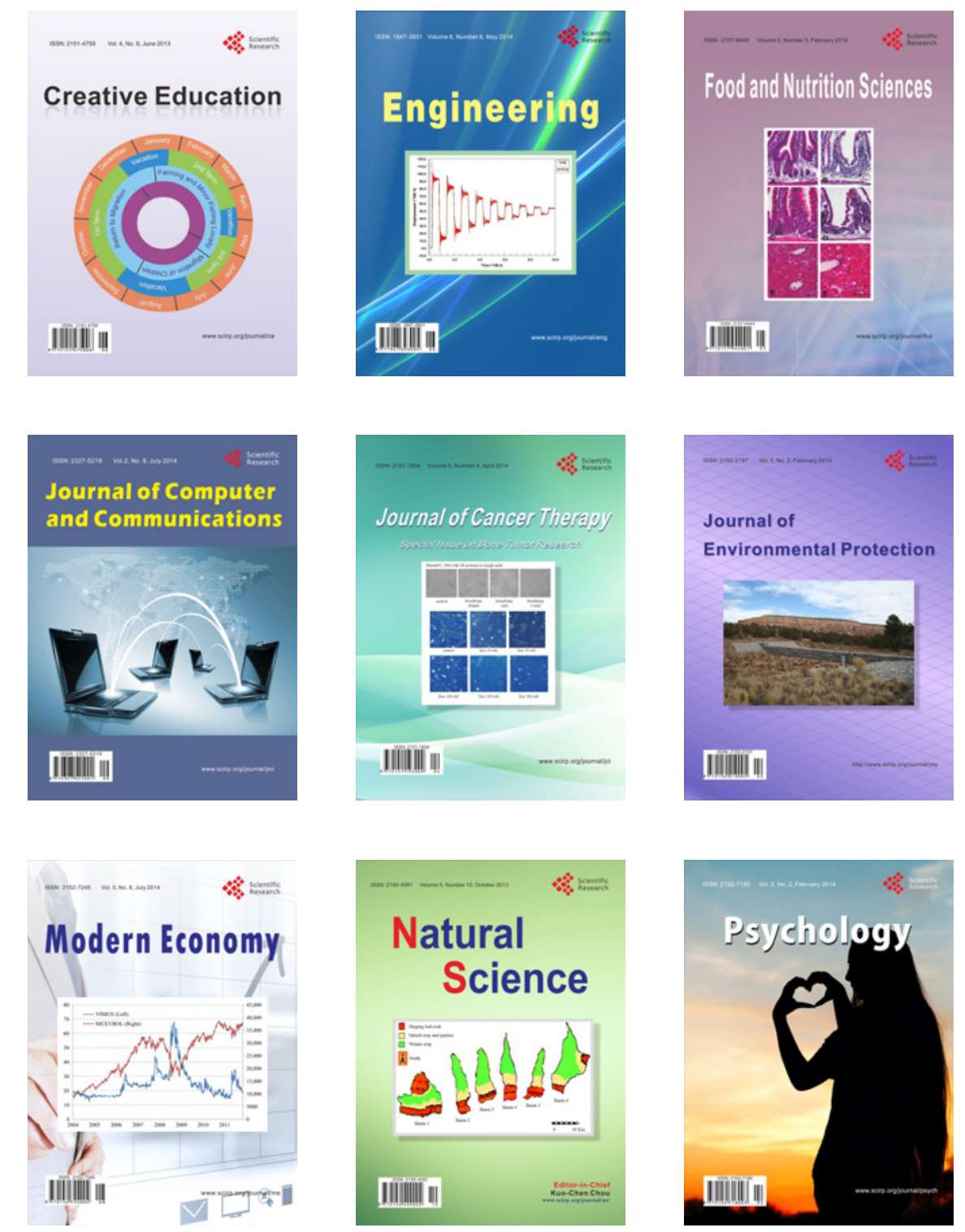